\title{
Introducing fluid dynamics using dimensional analysis
}

\author{
Jens Højgaard Jensen ${ }^{\text {a) }}$ \\ IMFUFA and "Glass and Time," Department of Sciences, Roskilde University, DK-4000 Roskilde, Denmark
}

(Received 25 October 2012; accepted 21 June 2013)

\begin{abstract}
Many aspects of fluid dynamics can be introduced using dimensional analysis, combined with some basic physical principles. This approach is concise and allows exploration of both the laminar and turbulent limits - including important phenomena that are not normally dealt with when fluid dynamics is first introduced as a precise mathematical discipline. In this paper, we use dimensional analysis to understand drag forces on bodies, flow speeds through tubes, and lift forces. (c) 2013 American Association of Physics Teachers.

[http://dx.doi.org/10.1119/1.4813064]
\end{abstract}

\section{INTRODUCTION}

In this article, we describe a method, using dimensional analysis, for introducing fluid dynamics to undergraduate physics students. Dimensional analysis, which emphasizes thinking more than memorizing, is not commonly used in introductory textbooks ${ }^{1-6}$ when discussing fluids, even though such as approach facilitates a clearer understanding of the effects of turbulence when considering drag and lift forces, and flow resistance in tubes.

Fluid dynamics is a huge and difficult subject, with a long history, not only in the field of physics but also in the fields of mathematics and engineering. In spite of this, a complete understanding of turbulence remains an unsolved problem, as several famous physicists have noted. ${ }^{7}$

Turbulence is common in daily life. The transition from laminar to turbulent flow is seen, for example, in smoke rising from a cigarette. Turbulence affects the air resistance acting on a bicyclist ${ }^{8}$ and on falling objects such as the coffee filters or muffin forms often used in physics teaching. ${ }^{9}$ In these cases, the air resistance is proportional to the square of the object's velocity, and this quadratic dependence on velocity, in incompressible fluids, is connected to turbulence. Nevertheless, in physics instruction drag forces are often assumed to be linear in the velocity (as is the case for laminar flow) rather than quadratic, even though the latter is more commonly encountered.

Within the paradigm of fluid dynamics as an exact mathematical discipline, drag forces proportional to velocity (Stokes' law) and other phenomena (e.g., Poiseuille's law for the flow speed through tubes) in the laminar flow regime can be derived. But due to the nonlinearity of the fundamental equations, this approach does not give results in the turbulent limit. In such situations dimensional analysis is especially useful, in that much of the behavior can be revealed without actually solving the nonlinear equations.

In Sec. II, we give a brief introduction to dimensional analysis, meant for readers unfamiliar with the method. In Secs. III and IV, we estimate drag forces on bodies and flow speeds through tubes in both the turbulent and laminar limits using dimensional analysis combined with work/energy-flow arguments. Instead of comparing the relative strengths of the terms (with dimensions of force) in the Navier-Stokes' equation, as is usually done in introductory textbooks on fluid dynamics, we ask whether the work done by driving forces is converted primarily into thermal energy or primarily into macroscopic kinetic energy. In Sec. V, we calculate lift forces in both the turbulent and laminar limits using dimensional analysis combined with force/momentum-flow arguments. In Sec. VI, we discuss how the usual mathematical way of presenting fluid dynamics has overshadowed the more intuitive approach of Secs. III-V. Finally, in Sec. VII, the pros and cons of the approach are discussed.

\section{DIMENSIONAL ANALYSIS}

In recognition of the centenary of its introduction, we will use the Bohr model of the hydrogen atom as an example to introduce the method of dimensional analysis.

When presenting his model in 1913, Niels Bohr gave a dimensional analysis argument in the introduction of his article. ${ }^{10}$ The classical mechanical problem of calculating the orbits of the electron around the proton involves only two input variables: the mass of the electron and the constant $k_{C}=e^{2} / 4 \pi \epsilon_{0}$ that determines the strength of the force between the two particles. But these two input variables cannot be combined into a physical quantity with dimensions of length, needed to explain the size of the atom. On the other hand, when Planck's constant is introduced as a third variable, dimensional analysis gives a characteristic length-the Bohr radius - that agrees with the known order of magnitude of the size of the atom. Let us see how.

We stress that the symbols used in physics formulas refer to physical quantities - not merely to the numerical values of these quantities in a particular system of units. For example, if we write $d_{1}=2 d_{2}$, to indicate that one distance is twice as large as another, we are making a statement that is independent of the particular units and numbers used to specify $d_{1}$ and $d_{2}$.

We also note that it is not possible to add quantities of different dimension-for example, a length and a mass. On the other hand, quantities with different dimensions can be multiplied and divided to obtain quantities with new dimensions (for example, when we calculate a density from a mass divided by a length cubed). In general, if a physical formula expresses some output quantity $Q_{1}$ in terms of several input quantities $Q_{2}, Q_{3}, \ldots$, then this formula must take the form

$$
Q_{1}=g \cdot Q_{2}^{\alpha} \cdot Q_{3}^{\beta} \cdot Q_{4}^{\gamma} \ldots
$$

where the powers $\alpha, \beta, \gamma, \ldots$ yield the correct dimensions for $Q_{1}$ and the dimensionless factor $g$ can be either a pure number or a function of dimensionless power-law combinations of $Q_{2}, Q_{3}, \ldots$, if such dimensionless combinations exist. ${ }^{11}$

Returning to the Bohr atom, we denote the Bohr radius $a_{0}$, the mass of the electron $m_{e}$, and write the Coulomb force between the electron and the proton as $k_{C} / r^{2}$. We will use square brackets to denote the dimensions of a quantity, and 
the symbols $L, T$, and $M$ to denote the fundamental mechanical dimensions length, time, and mass, respectively. We can then write $\left[a_{0}\right]=L,\left[m_{e}\right]=M$, and $\left[k_{C}\right]=M \cdot L^{3} \cdot T^{-2}$ (note that the final expression is equivalent to force times $L^{2}$ ).

Now suppose that $a_{0}$ were a function of only $m_{e}$ and $k_{C}$. Then, following the form of Eq. (1), we could write

$$
a_{0}=g \cdot\left(m_{e}\right)^{\alpha} \cdot\left(k_{C}\right)^{\beta}
$$

for some $\alpha$ and $\beta$. Taking the dimensions of both sides of this equation gives

$$
L=M^{\alpha} \cdot\left(M \cdot L^{3} \cdot T^{-2}\right)^{\beta}=M^{\alpha+\beta} \cdot L^{3 \beta} \cdot T^{-2 \beta} .
$$

Since none of the fundamental dimensions $L, T$, and $M$ can be written in terms of the others, the powers of each dimension must match up separately between the two sides. But this is impossible - matching powers of $L$ implies $\beta=1 / 3$, while matching powers of $T$ implies $\beta=0$.

Bohr's dimensional analysis thus shows that classical mechanics cannot produce a characteristic atomic length. But including Planck's constant (divided by $2 \pi$ ), which has dimension $[\hbar]=M \cdot L^{2} \cdot T^{-1}$, changes the situation. Equations (2) and (3) are then replaced by

$$
a_{0}=g \cdot\left(m_{e}\right)^{\alpha} \cdot\left(k_{C}\right)^{\beta} \cdot \hbar^{\gamma}
$$

and

$$
\begin{aligned}
L & =M^{\alpha} \cdot\left(M \cdot L^{3} \cdot T^{-2}\right)^{\beta} \cdot\left(M \cdot L^{2} \cdot T^{-1}\right)^{\gamma} \\
& =M^{\alpha+\beta+\gamma} \cdot L^{3 \beta+2 \gamma} \cdot T^{-2 \beta-\gamma} .
\end{aligned}
$$

Equating the powers of the fundamental dimensions now gives

$$
\begin{aligned}
M: & 0=\alpha+\beta+\gamma, \\
L: & 1=3 \beta+2 \gamma \\
T: & 0=-2 \beta-\gamma,
\end{aligned}
$$

and these equations have the unique solution $\alpha=-1$, $\beta=-1$, and $\gamma=2$. Similarly, searching for combinations of $m_{e}, k_{C}$, and $\hbar$ with dimension $L^{0} T^{0} M^{0}$ gives the unique solution $\alpha=\beta=\gamma=0$, implying that the factor $g$ in Eq. (4) has to be a pure number. Therefore, from dimensional reasoning we conclude that a theory of atoms built on the input variables $m_{e}, k_{C}$, and $\hbar$, independent of other assumptions, results in a characteristic atomic length given by

$$
a_{0}=\text { pure number } \cdot \frac{\hbar^{2}}{m_{e} k_{C}} \text {. }
$$

This expression happens to agree with the textbook formula for the Bohr radius if the pure number is chosen to be 1 .

Dimensional analysis thus played a role in the birth of atomic physics and quantum mechanics. Of course, the value (in this case 1 ) of the pure number cannot be found using dimensional analysis. But aside from this pure number, the Bohr radius can be found using dimensional analysis without reference to a developed model or theory. Any model or theory that uses $m_{e}, k_{C}$ and $\hbar$ as input variables will imply Eq. (7). Thus, it is not surprising that the correct theory (Schrödinger's equation) gives the same expression.
It is not common for physicists to follow Niels Bohr and present dimensional analysis in their formal writings. But dimensional analysis is a powerful tool that physicists often use before developing a more formal line of reasoning. Solving problems using dimensional analysis is also a good approach for teaching students how to think like a physicist. $^{11}$

\section{DRAG}

We now turn to fluid dynamics, and use dimensional analysis to calculate the drag force on a body moving through a fluid. For motion through air, drag is often called air resistance. But the formulas we derive below are valid for the motion of bodies in both gases and liquids. We consider the steady motion of a body pulled though a fluid, as sketched in Fig. 1. The drag $D$ is the force exerted by the fluid in the direction opposite to the motion. If the motion is steady, the drag has the same magnitude as the pulling force.

The first thing to do is to ask which physical quantities the drag can depend on. The pulling force and the drag depend on the velocity $v$ of the body; they also depend on the shape of the body, and on a length $r$ characterizing the size of the body. We further expect the drag to depend on the density $\rho$ of the fluid. And we must expect the drag to depend on the internal "friction" or "stickiness" within the fluid, characterized by its viscosity $\eta$.

We might at first think that the mass or density of the body is a relevant physical quantity, but there is no physical mechanism for mass to affect the pulling force unless the body is accelerated. Or we might think that the compressibility of the fluid is relevant - and in fact it is, if $v$ is near to the speed of sound. We therefore restrict ourselves to velocities small compared to the speed of sound, which is equivalent to assuming that the fluid is incompressible. This leaves us with $v, r, \rho, \eta$, and dimensionless quantities characterizing the shape of the body, as input variables for $D$. The formula for $D$, following Eq. (1), must therefore take the form

$$
D=g \cdot v^{\alpha} \cdot r^{\beta} \cdot \rho^{\gamma} \cdot \eta^{\delta}
$$

with $g$ depending on the shape of the body and possibly on dimensionless combinations of the other variables.

We seek values of $\alpha, \beta, \gamma$, and $\delta$ that give the same dimensions on both sides of Eq. (8). To find the dimensions of $\eta$, we imagine a plate on the top of a layer of fluid resting on a

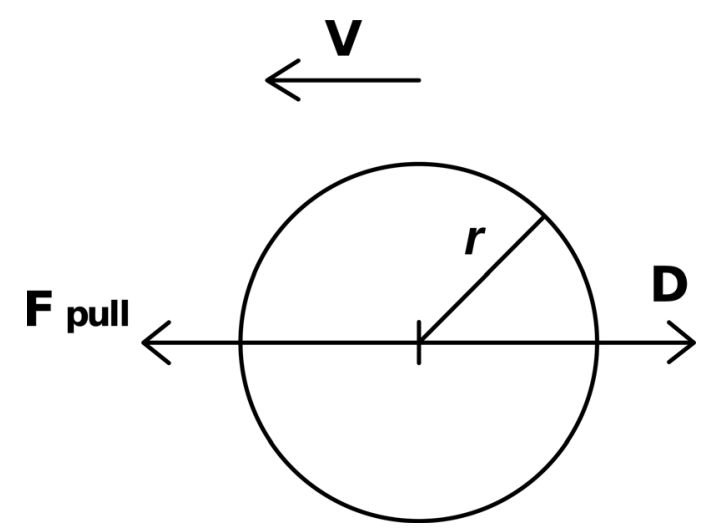

Fig. 1. Drag force on a body pulled through a fluid. Here $\mathbf{F}_{\text {pull }}$ is the pulling force, $\mathbf{D}$ the drag force, $\mathbf{v}$ the velocity of the body, and $r$ a length characterizing the size of the body. 
horizontal plane. Acting on the plate with a horizontal force causes the plate to move along with the top of the fluid layer, while the bottom of the layer remains at rest, "stuck" to the surface below. The greater the viscosity, the more force is required, per unit area of the plate, to produce a given velocity gradient (change of velocity per unit height). The viscosity coefficient $\eta$ is therefore defined as

$$
\eta=\frac{\text { force } / \text { area }}{d v / d z}
$$

where $z$ is the vertical direction. From this definition it is straightforward to show that $[\eta]=M \cdot L^{-1} \cdot T^{-1}$.

The dimensions of the remaining quantities in Eq. (8) are familiar, so we can now take the dimensions of both sides of that equation to obtain

$$
M^{1} \cdot L^{1} \cdot T^{-2}=M^{\gamma+\delta} \cdot L^{\alpha+\beta-3 \gamma-\delta} \cdot T^{-\alpha-\delta} .
$$

Equating the powers of the base dimensions on the two sides of the equation gives

$$
\begin{array}{ll}
M: & 1=\gamma+\delta, \\
L: & 1=\alpha+\beta-3 \gamma-\delta, \\
T: & -2=-\alpha-\delta .
\end{array}
$$

Because there are three equations and four unknowns, dimensional analysis does not uniquely determine the formula for $D$. However, we can use these three equations to eliminate $\alpha, \beta$, and $\gamma$ in Eq. (8), which then becomes

$$
D=g \cdot v^{2-\delta} \cdot r^{2-\delta} \cdot \rho^{1-\delta} \cdot \eta^{\delta}=g \cdot \rho r^{2} v^{2} \cdot\left(\frac{\eta}{\rho r v}\right)^{\delta}
$$

in which only $\delta$ and $g$ remain to be determined. The quantity $\eta / \rho r v$ is dimensionless; it is the inverse of Reynolds number $\operatorname{Re} \equiv \rho r v / \eta$.

The unknown $g$ is either a pure number or a function of dimensionless combinations of $v, r, \rho$, and $\eta$. If we modify the preceding analysis to seek a dimensionless combination of these variables, we find that the only possibilities are $(\rho r v / \eta)^{\alpha}=\operatorname{Re}^{\alpha}$. We can therefore rewrite Eq. (12) as

$$
D=\rho r^{2} v^{2} \cdot f(\mathrm{Re}),
$$

where $f$ is an unknown function of Re. This is the usual formula for the drag, found in textbooks in fluid dynamics. The function $f(\mathrm{Re})$ depends on the shape of the body and can be very complex. Much effort is invested in understanding this function in fluid dynamics.

Here, however, we will skip the formal reasoning and continue with a more intuitive physical approach. In general, both thermal energy and macroscopic kinetic energy are created when a body is pulled through a fluid. But if we restrict ourselves to either the limit where the velocity of the body is very small, or the limit where the velocity of the body is very large, we can be more explicit. ${ }^{12}$ Our guide is the question: Is the work done by the pulling force converted primarily into thermal energy or primarily into macroscopic kinetic energy of the fluid?

If the velocity of the body is sufficiently small, we expect a steady, laminar flow around the body. In the reference frame of the body, the velocity at every location is constant and therefore the macroscopic kinetic energy is constant. This means that the work done by the pulling force must go entirely into thermal energy, caused by the internal friction in the fluid. In this limit, the drag force will therefore depend on the viscosity of the fluid but not on its density, because the density enters into only the macroscopic kinetic energy.

Thus, in the low-velocity limit Eq. (8) is replaced by the simpler formula

$$
D=g \cdot v^{\alpha} \cdot r^{\beta} \cdot \eta^{\gamma}
$$

and taking the dimension of both sides gives

$$
M^{1} \cdot L^{1} \cdot T^{-2}=M^{\gamma} \cdot L^{\alpha+\beta-\gamma} \cdot T^{-\alpha-\gamma},
$$

so that Eqs. (11) are replaced by

$$
\begin{array}{ll}
M: & 1=\gamma, \\
L: & 1=\alpha+\beta-\gamma, \\
T: & -2=-\alpha-\gamma .
\end{array}
$$

These equations have the unique solution $\alpha=\beta=\gamma=1$. Moreover, there is no combination of $v, r$, and $\eta$ that is dimensionless, so $g$ is simply a number. Our formula for the drag force in this limit therefore becomes

$$
D=\text { pure number } \cdot \eta r v,
$$

where the pure number depends of the shape of the body. For a sphere of radius $r$, the number happens to be $6 \pi$ and Eq. (17) is known as Stokes' law.

In the opposite limit, where the velocity is sufficiently large, we expect a turbulent wake behind the body. When pulling the body through the fluid, we continuously produce new macroscopic kinetic energy in the wake. At sufficiently high velocities, we will assume that the work done by the pulling force is converted predominantly into macroscopic kinetic energy in the wake. The macroscopic kinetic energy of a fluid with a given flow pattern depends of the density of the fluid, but not on its viscosity. Although the viscosity of the fluid determines how the macroscopic kinetic energy in the wake is transformed into thermal energy in the long run, the immediate production of thermal energy in the fluid can be neglected. In this case, we therefore expect the pulling force (and the oppositely directed drag of equal magnitude) to be independent of viscosity, determined only by shape, $v$, $r$, and $\rho$. Again we now have only three input variables, besides the dimensionless shape variables, so the dependence of $D$ on the three variables is determined uniquely. By an argument entirely analogous to those given previously, we find

$$
D=\text { pure number } \cdot \rho r^{2} v^{2} .
$$

This formula describes the air resistance acting on a bicyclist, $^{8}$ a moving automobile, ${ }^{8}$ and the falling coffee filters found in school physics experiments.

Comparing Eq. (17), valid for small velocities, with Eq. (13), valid in general, we conclude that $f(\mathrm{Re})$ is proportional to $(\mathrm{Re})^{-1}$ at small velocities. Similarly, by comparing Eq. (18), valid for large velocities, with Eq. (13), we find $f(\mathrm{Re})=$ constant. Since $f(\mathrm{Re})$ is a function of $\mathrm{Re}$, and not of $v$, we should rather state that $f(\mathrm{Re}) \propto(\mathrm{Re})^{-1}$ at small values of $\mathrm{Re}$, and that $f(\mathrm{Re})$ is independent of $\mathrm{Re}$ at large values of 
Re. In the reasoning above $r, \eta$, and $\rho$ were assumed constant and only $v$ varied. But the more general statements are that Eq. (17) is valid in the limit of small Reynolds numbers, and Eq. (18) is valid in the limit of large Reynolds numbers. How small or large the Reynolds numbers should be in order to make Eqs. (17) and (18) reliable depends on the shape of the body. In the cases of bicycling, car driving, and falling coffee filters, Eq. (18) is valid and $D$ is thus independent of Re. This means that the assumption of $D$ being independent of $\eta$ is justified in these cases. In general, at a given Re, Eq. (18) is more valid for not-very-streamlined bodies, where the cross section of the wake is determined by the cross section of the body, not by the detailed streaming of the fluid around the body.

\section{FLOW}

Using the same methods as above, we now treat fluid flow through a tube in the two limits. We imagine a fluid being driven through a tube by a pressure difference between its ends, as sketched in Fig. 2. As in the case of drag, we focus on whether thermal energy or macroscopic kinetic energy is created in the tube as the fluid is driven through it.

Our goal is to find a formula for the volume of fluid per unit time flowing through the tube, $Q$. The flow rate $Q$ must depend on the shape and the characteristic length $r$ of the cross section of the tube. It depends on the pressure difference between the ends of the tube $\Delta P$ and on the length of the tube $\ell$ in the combination $\Delta P / \ell$ because the flow of fluid will be unaltered when doubling both $\Delta P$ and $\ell$. In general, we expect $Q$ to depend on both the viscosity $\eta$ and the density $\rho$ of the fluid. As in the preceding section, this leaves one input variable too many to determine the desired formula by dimensional analysis. Thus, we will restrict ourselves to the two limits where the Reynolds number is either very small or very large. The relevant velocity entering the estimate of the Reynolds number $(\operatorname{Re}=\rho r v / \eta)$ is the average flow velocity, given by $Q$ divided by the cross-sectional area of the tube.

Consider first the limit where Re is small. Here we expect a laminar and steady flow through the tube. The macroscopic kinetic energy of the fluid is thus the same at the end as at the beginning of the tube. This means that the work done in pressing the fluid through the tube goes entirely into heating the fluid. This heating depends on the fluid's viscosity but not on its density. We thus expect $Q$ to be determined by $r$, the cross section shape, $\Delta P / \ell$, and $\eta$. Because $[Q]=L^{3} \cdot T^{-1}$ and $[\Delta P / \ell]=M \cdot L^{-2} \cdot T^{-2}$, the only combination of these quantities that has the correct dimensions is $(\Delta P / \ell) \cdot r^{4} / \eta$. No dimensionless quantities can be made from $\Delta P / \ell, r$, and $\eta$, so the formula for $Q$ at small Reynolds numbers is

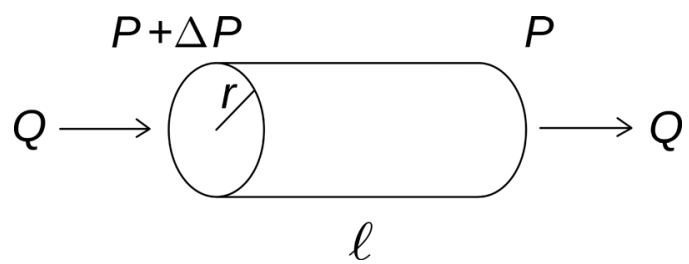

Fig. 2. Fluid flow through a tube due to a pressure difference. Here $Q$ is the volume of fluid driven through the tube per unit time, $r$ a characteristic cross sectional width, $\ell$ the length of the tube, and $P$ the pressure.

$$
Q=\text { pure number } \cdot\left(\frac{\Delta P}{\ell}\right) \cdot \frac{r^{4}}{\eta}
$$

Inserting $\pi / 8$ for the number gives Poiseuille's law for flow through a tube with a circular cross section of radius $r$.

Next consider the limit where the Reynolds number is large. Here, we expect that the interaction with the walls of the tube causes turbulent motion of the fluid, with increasing velocity components perpendicular to the stream direction as the fluid moves through the tube. The fluid thus leaves the tube with larger macroscopic kinetic energy per unit volume than when it entered. The work done by the pressure difference forcing the fluid through the tube is converted into macroscopic kinetic energy in the fluid, which depends on the fluid's density $\rho$, but not on its viscosity. In this limit, we therefore expect the flow rate $Q$ to be determined by $r$, cross section shape, $\Delta P / \ell$, and $\rho$. Dimensional analysis for the desired formula for $Q$ then results in

$$
Q=\text { pure number } \cdot\left(\frac{\Delta P}{\ell} \cdot \frac{r^{5}}{\rho}\right)^{1 / 2}
$$

This formula applies for tubes with not-too-smooth internal surfaces, e.g., cast-iron tubes. The formula is seldom derived in physics textbooks; I only found it reported as an empirical result in specialized textbooks in fluid mechanics. ${ }^{13}$

Equation (20) has an interesting consequence. Imagine two containers partly filled with the same fluid connected to each other by a tube at their bottoms. Suppose the surfaces of the fluid in the two containers are initially at different heights, thereafter gradually reaching the same level due to flow, driven by gravity, through the tube. It follows from Eq. (20) that the way this takes place, at large Reynolds numbers, is independent of the fluid: $Q$ is independent of $\rho$ whenever $\Delta P$ is proportional to $\rho$, as in this case for fluids in a gravitational field. Thus, the way potential energy is converted into turbulent macroscopic kinetic energy does not depend on density, just as the conversion of potential energy into kinetic energy during free fall does not depend on mass.

Summarizing, we have derived formulas for both drag and flow, in both the turbulent and the laminar limits. This was accomplished using dimensional analysis combined with physical reasoning about the transformation of work into primarily either thermal energy or macroscopic kinetic energy. In Sec. V, we treat lift forces in general, using dimensional analysis combined with physical reasoning about the flow of momentum in the fluid passing a body.

\section{LIFT}

Why can dust and sand grains be lifted by the wind, given the fact that they afterward fall to the ground again and thus are not able to float? What is the minimal wind velocity needed to lift the grains from the ground? Let us try to answer this question as an illustration of fluid lift problems in general.

Figure 3 suggests that the lift force $L$ on the grain, due to its interaction with the air passing by the grain, derives from the change of the direction of motion of the air during the passage. According to Newton's third law, the grain must cause a force on the air in the opposite direction and of the same magnitude as the lift force exerted by the air on the grain. According to Newton's second law, an unbalanced 


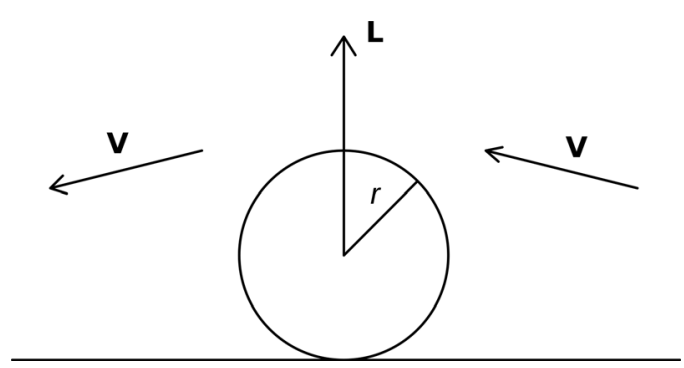

Fig. 3. Lift force on a sand grain. Here $\mathbf{L}$ is the lift force, $\mathbf{v}$ the velocity of the wind, and $r$ a characteristic length.

force on the air is accompanied by an equal change in its momentum per unit time. The lift $L$ can thus be evaluated by calculating the vertical momentum change per unit time of the air passing the particle.

Unlike the drag force, the lift force is not intuitively related to friction and the viscosity of the air. We assume that the streaming pattern around the grain is determined by the geometry, the velocity of the air, and the density of the air. Furthermore, we assume that the magnitude of the viscosity influences only the thickness of a thin boundary layer, caused by the condition of zero velocity at the boundary. In this case, the vertical momentum change of the air per unit time depends only on the shape of the grain, the size of the grain $r$, the density of air $\rho_{\text {air }}$, and the velocity of the wind $v$. Therefore the lift force, by dimensional analysis, must be

$$
L=\text { pure number } \cdot \rho_{\text {air }} \cdot r^{2} v^{2},
$$

where the dimensionless number depends on the shape of the grain.

The grains are lifted from the ground when the lift force exceeds the gravitational force minus the buoyant force. The critical wind velocity $v_{\text {crit }}$ for sandstorms is therefore given by

$$
\rho_{\text {air }} r^{2} v_{\text {crit }}^{2}=\text { pure number } \cdot\left(\rho_{\text {sand }}-\rho_{\text {air }}\right) r^{3} g
$$

or

$$
v_{\text {crit }}=\text { pure number } \cdot \sqrt{r g\left(\frac{\rho_{\text {sand }}}{\rho_{\text {air }}}-1\right)},
$$

where $\rho_{\text {sand }}$ is the density of sand and $g$ is the strength of the gravitational field. The static buoyant force is included so that Eq. (23) also covers phenomena such as streaming water lifting sediments in rivers and the sea, where $\rho_{\text {water }}$ cannot be neglected compared to $\rho_{\text {sand }}$. Equation (23) is found in the literature on dynamics of sand, wind, and barchans. ${ }^{14}$

After being lifted, the grain has a symmetric stream pattern around it, with no resulting vertical momentum transfer between it and the air. The grain will thus fall to the ground again because of the absence of a lift force.

It is worth emphasizing that we assumed neither laminar nor turbulent flow when deriving Eq. (21). Contrary to the expressions for drag and flow, the same formula for lift applies in both the low-velocity and high-velocity limits.

\section{MATHEMATICS OVERSHADOWING PHYSICAL INTUITION}

The discipline of fluid dynamics has a long and impressive history. The master's thesis of Werner Heisenberg in 1923, at the Institute of Theoretical Physics in Munich, was in fluid dynamics with Arnold Sommerfeld as his supervisor. Fluid dynamics was considered an important part of theoretical physics in Germany at that time. In England the subject was studied in departments of applied mathematics. Whether fluid dynamics was characterized as theoretical physics or applied mathematics, it was approached as an exact mathematical discipline. Thus the first edition from 1879 of Horace Lamb's classical book about hydrodynamics $^{15}$ was entitled Treatise on the Mathematical Theory of the Motion of Fluids. It is a highly mathematical book, even though it was renamed Hydrodynamics in later editions.

In The Feynman Lectures on Physics, Richard P. Feynman ${ }^{16}$ divides fluid dynamics into "The Flow of Dry Water" and "The Flow of Wet Water." Dry water is his name for ideal fluids with no viscosity; wet water is the name for real fluids with viscosity. According to Feynman, "John von Neumann was well aware of the tremendous difference between what happens when you do not have the viscous terms and when you do, and he was also aware that, during most of the development of hydrodynamics until about 1900, almost the main interest was in solving beautiful mathematical problems with this approximation which had almost nothing to do with real fluids. He characterized the theorist who made such analyses as a man who studied 'dry water." The term dry water is thus originally due to von Neumann.

In his introduction to the flow of wet water, Feynman nevertheless follows the tradition and puts the emphasis on mathematical beauty. He ends up with the Navier-Stokes equation for an incompressible fluid in the form

$$
\frac{\partial \Omega}{\partial t}+\nabla \times(\Omega \times \mathbf{u})=\frac{1}{\operatorname{Re}} \cdot \nabla^{2} \Omega ; \quad \Omega=\nabla \times \mathbf{u} .
$$

Here distances, velocities, and time are measured in units of $r, v$ and $r / v$, with $r$ being a characteristic length and $v$ a characteristic velocity of a problem considered, $\mathrm{Re}$ being the Reynolds number. Equation (24) is not only a beautiful mathematical result but also very useful because it proves that geometrically similar problems have the same relative velocity field $\mathbf{u}$, as long as their Reynolds numbers are identical. This theorem justifies applying the results of windtunnel measurements on small-scale airplanes or modelbasin results on scale-model boats to full-scale objects.

For both mathematical and engineering reasons, the tradition in fluid mechanics is therefore to consider different physical quantities as functions of the Reynolds number. The drag force on a body pulled through a fluid is in general expressed in the form of Eq. (13). The function $f(\operatorname{Re})$ is called the drag coefficient. In most textbooks in fluid mechanics, empirically found drag coefficients for different shapes are presented as functions of Re. In the limit of small Reynolds numbers - that is, the limit of small velocities if $r$, $\rho$, and $\eta$ are held fixed- $f(\mathrm{Re})$ is found to be inversely proportional to Re, giving Eq. (17), Stokes' law.

In the limit of large Reynolds numbers (large velocities), it is, however, not customary to report $f(\mathrm{Re})$ to be a constant function, independent of Re, as in Eq. (18). Lautrup has written an excellent book introducing fluid dynamics within the mathematical tradition, where he merely mentions that the drag becomes independent of the Reynolds number at large Reynolds numbers. ${ }^{17}$ Landau and Lifshitz argue that the drag 
coefficient for a flat disk held perpendicular to the flow direction must be independent of Re in the high velocity limit, but this is again mentioned only as an aside. ${ }^{18}$ In general, the focus in the literature is on Eq. (13) with its four input variables, not allowing $D$ to be determined by dimensional analysis. Authors rarely give arguments for $D$ being independent of viscosity at high velocities and therefore having only three input variables and being given by dimensional analysis as Eq. (18). ${ }^{19}$ As mentioned, this leaves many daily experiences unexplained.

What is the explanation for the lack of attention to drag forces proportional to $v^{2}$, despite their frequent appearance in nature and in everyday life? The best explanation I can give is that the prevailing paradigm in fluid mechanics, developed by mathematicians on the theoretical side, and engineers on the practical side, expresses everything as a function of the Reynolds number, which overshadows the more intuitive physical arguments leading to Eq. (18).

Likewise, when dealing with flow through a pipe, the paradigm seems to have overshadowed the arguments leading to Eq. (20).

\section{DISCUSSION}

Of course the high-velocity limit with turbulence-or more correctly, the limit of high Reynolds numbers-is difficult to tackle compared to the limit of small velocities and laminar flow. Experience shows that laminar flow, and consequently the expressions in Eqs. (17) and (19) for $D$ and $Q$, is always reached eventually as the velocity is reduced. However, the expressions in Eqs. (18) and (20) for $D$ and $Q$ cannot always be reached when the velocity is increased, as we shall now see.

First consider drag. Here, Eq. (18) for a flat disk perpendicular to the direction of the flow is usually reached at nottoo-large velocities, long before the assumption of incompressibility breaks down at velocities comparable to the speed of sound. But this is not the case for very streamlined objects such as wings of birds and airplanes. What is wrong with the argument in Sec. III, leading to Eq. (18)? The argument was that the work done by the pulling force to move a body through a fluid, in the high-velocity limit, results mainly in macroscopic kinetic energy in the turbulent wake behind the body. And because the macroscopic kinetic energy depends on $\rho$, not on $\eta$, the same must be true for the pulling force and thus the drag.

The shortcoming of this argument is that, although only $\rho$ is needed to express the macroscopic kinetic energy in the wake, the macroscopic kinetic energy in the wake also depends on $\eta$ because $\eta$ determines how the wake is created at the body. In a fluid without viscosity there would not be a turbulent wake. For many sorts of bodies the influence of $\eta$ is so complicated that the drag force, over a certain range of the Reynolds number, decreases with increasing velocity, because of a narrowing of the wake with increasing velocity.

The circumstance where the argument leading to Eq. (18) is justified is when the turbulent wake is determined by geometry alone, as in the case of the flat disk perpendicular to the flow. Although viscosity in the fluid is here also needed to create a turbulent wake, the magnitude of the viscosity does not matter if $\eta$ is larger than a certain minimum size. An analog is a problem involving static friction. A minimal static friction coefficient may be needed, but if this condition is satisfied, the solution does not depend on the actual value of the friction coefficient. As mentioned, many daily phenomena act more like the flat disk than like a streamlined body: turbulent wakes are, in essence, determined by the cross section to the flow, not by the magnitude of the viscosity.

For flow through a pipe, Eq. (20) has been experimentally verified for rough pipes, but not for smooth pipes. ${ }^{13}$ The less rough the pipe is, the higher is the Reynolds number that must be exceeded before the formation of turbulence is independent of the viscosity, corresponding to Eq. (20).

In the derivations of Eqs. (19) and (20), the pressure gradient was assumed constant along the pipe. The quantities $\Delta P$ and $\ell$ appeared only in the combination $\Delta P / \ell$ in the dimensional analysis. Due to translational symmetry, this assumption is valid in the laminar limit. However, in the turbulent limit, with increasing turbulent macroscopic kinetic energy along the pipe, the validity of this assumption is not obvious. In practice, it seems to be a valid approximation. ${ }^{13}$ Theoretically, an unknown function of $\ell / r$ would have to be substituted for the pure number in Eq. (20) if $\Delta P$ and $\ell$ are considered independent input variables.

Explanations of lift on airplanes were discussed some time ago in this journal by Weltner. ${ }^{20}$ He argued that an explanation based on repulsive forces is easier to assimilate, more powerful, and better related to the basic principles of mechanics than the frequently used explanation using Bernoulli's equation. Furthermore, he argued that the explanation based on Bernoulli's equation has fundamental drawbacks and is based on incomplete reasoning. Weltner argued "that the high streaming velocity at the upper side of the aerofoil is not the reason for the low pressure. To the contrary, the low pressure generated by the aerofoil is the reason for the high streaming velocity."

The treatment of lift in Sec. V, which relates the lift to repulsive forces and not to Bernoulli's equation, is in accordance with Weltner. Equation (21) is relevant not only for the lift on sand grains but also for the lift on airplanes, even though an airplane wing, unlike a sand grain, is not characterized by just one length. If we assume that the flow pattern around the wing is given by geometry and is independent of the value of the viscosity of the air, dimensional reasoning tells us again that $\rho_{\text {air }}$ and $v$ go into the expression for the lift with the factor $\rho_{\text {air }} v^{2}$, as in Eq. (21).

The assumption of a solely geometrically determined flow pattern is common. It was also made by Weltner for calculating the momentum transfer to the air from a steady laminar flow around an airplane wing. The assumption is, however, equally valid when the flow is turbulent. In both the turbulent and the laminar limits, the value of viscosity merely affects the thickness of a boundary layer, which can be treated as a part of the body to be lifted. Between these two limits, the ways in which vortices go into the flow depend in general on the values of the viscosity and the Reynolds number. Thus, the number in Eq. (21) is not merely a constant but a function Reynolds number. It is only in the two limits that dimensional analysis leads to a $v^{2}$ dependence of the lift.

In summary, drag, flow, and lift can all be explained qualitatively in an introductory physics course by relating these phenomena to the basic principles of physics. In the case of lift forces, the basic principles are Newton's second and third laws. For drag forces and flow through a pipe, the basic principle is the work-energy theorem, with the energy produced 
becoming primarily either macroscopic kinetic energy or thermal energy. Through the use of dimensional analysis these explanations can be developed to give quantitative results, both in the laminar and the turbulent limits. Because the results in the turbulent limit, not usually presented in physics textbooks, are in accordance with many daily experiences, examining such cases via dimensional analysis provides a good introduction to fluid dynamics before drowning in the mathematical details and intermediate-Reynolds-number phenomena relevant for more-or-less streamlined bodies and smooth tubes.

\section{CONCLUDING REMARKS}

The mathematical and experimental complexities of the huge subject of fluid dynamics have, in the physics teaching tradition, led to two alternatives: either the subject is mostly avoided or it is taught as an exact mathematical discipline. In both cases, common phenomena such as drag forces proportional to the velocity squared are not discussed.

Fortunately, the observation that mathematics can cast a shadow over physical intuition is not common in physics. In fact, mathematical thinking has been a characteristic aspect of physics for 400 years, ever since Galileo stated that the book of nature is written in the language of mathematics. In particle mechanics, for instance, precise mathematical thinking may be essential. ${ }^{21}$ Nevertheless, beginning with Maxwell the letter symbols in the mathematical equations of physics have gradually come to be understood as representing physical quantities, not merely pure numbers, thus transforming theoretical physics from being understood as number calculus to quantity calculus. ${ }^{22}$ This development has made dimensional analysis another important aspect of theoretical physics.

We conclude by noting that dimensional analysis is a powerful tool in physics; introducing fluid dynamics through dimensional analysis, combined with basic physical principles, makes it possible in an introductory physics course to deal with drag, flow, and lift, in both the laminar and turbulent limits.

\section{ACKNOWLEDGMENTS}

The author wishes to thank Jeppe Dyre, Nicholas Baily, Torben Rasmussen, Poul Winther Andersen, Tage Cristensen, Mikkel Jensen, and the reviewers for their assistance and helpful suggestions. The center for viscous liquid dynamics "Glass and Time" is sponsored by the Danish National Research Foundation (DNRF 61).

${ }^{\text {a)} E l e c t r o n i c ~ m a i l: j h j @ r u c . d k ~}$

${ }^{1}$ H. C. Ohanian and J. T. Markert, Physics for Engineers and Scientists, 3rd ed. (Norton, New York, 2007).

${ }^{2}$ P. A. Tipler and G. Mosca, Physics for Scientists and Engineers, 6th ed. (Freeman, New York, 2008).

${ }^{3}$ H. D. Young and R. A. Freedman, University Physics, 13th ed. (Pearson Addison-Wesley, New York, 2012).

${ }^{4}$ R. D. Knight, Physics for Scientists and Engineers, 3rd ed. (Pearson, New York, 2013).

${ }^{5}$ D. C. Giancoli, Physics for Scientists and Engineers, 4th ed. (Pearson, London, 2008).

${ }^{6}$ J. Keller, W. E. Gettys and M. J. Skove, Physics: Classical and Modern, 2nd ed. (McGraw-Hill, 1993).

${ }^{7}$ See, for example, the Wikipedia article "Turbulence," < http://en.wikipedia.org/wiki/Turbulence>, which (as of 14 June 2013) quotes Feynman, Heisenberg, and Lamb on the importance and difficulty of understanding turbulence.

${ }^{8}$ Since $\eta / \rho$ is $15 \times 10^{-6} \mathrm{~m}^{2} / \mathrm{s}$ for air at room temperature, typical values such as $r=1.5 \mathrm{~m}$ and $v=36 \mathrm{~km} / \mathrm{h}=10 \mathrm{~m} / \mathrm{s}$ give a Reynolds number of $10^{6}$.

${ }^{9}$ Searching in Google on "physics lab falling coffee filters" gives 130,000 results.

${ }^{10} \mathrm{~N}$. Bohr, "On the constitution of atoms and molecules, part I," Philos. Mag. 26, 1-25 (1913).

${ }^{11}$ For a further introduction to dimensional analysis, see, for example, $\mathrm{T}$. Misic, M. Najdanovic-Lukic, and L. Nesic, "Dimensional analysis in physics and the Buckingham theorem," Eur. J. Phys. 31, 893-906 (2010); and the classic, P. W. Bridgman, Dimensional Analysis (Yale U.P., New Haven, 1922).

${ }^{12}$ The velocity still has to be small compared to the velocity of sound in the fluid.

${ }^{13}$ R. A. Granger, Fluid Mechanics (Dover, New York, 1995), p. 496.

${ }^{14}$ B. Andriotti, P. Claudin, and S. Douady, "Selection of dune shapes and velocities, Part 1: Dynamics of sand, wind and barchans," Eur. Phys. J. B 28, 321-339 (2002).

${ }^{15}$ H. Lamb, Hydrodynamics, 4th ed. (Cambridge U.P., Cambridge, 1916).

${ }^{16}$ R. P. Feynman, R. B. Leighton, and M. Sands, The Feynman Lectures on Physics (Addison-Wesley, Reading, MA, 1964), Vol. II, Chaps. 40 and 41.

${ }^{17}$ B. Lautrup, Physics of Continuous Matter, 2nd ed. (CRC Press, Taylor and Francis Group, London, 2011), p. 295.

${ }^{18}$ L. Landau and E. M. Lifshitz, Fluid Mechanics, 2nd ed. (Elsevier, Oxford, 2009), p. 182.

${ }^{19}$ An exception is the popular book A. H. Shapiro, Shape and Flow (Doubleday, Garden City, New York, 1961).

${ }^{20} \mathrm{~K}$. Weltner, "A comparison of explanations of the aerodynamic lifting force," Am. J. Phys. 55, 50-54 (1987).

${ }^{21}$ See, for example, J. H. Jensen, "Five ways of deriving the equation of motion for rolling bodies," Am. J. Phys. 80(12), 1073-1077 (2012).

${ }^{22} \mathrm{~J}$. de Boer, "On the history of quantity calculus and the international system," Metrologia 32, 405-429 (1995), is recommended for a brief presentation of the most important developments in the history of quantity calculus. 